abnormalities of brain development. Focal or multifocal noncystic WMI is the most prevalent pattern of brain injury in premature newborns (Hamrick SE et al. 2004, cited by authors).

A previous meta-analysis report from UCSF demonstrates that chorioamnionitis is a risk factor for cerebral palsy and/or cystic periventricular leukomalacia in the term and preterm neonate. (Wu YW, Colford JM Jr. JAMA 2000;284:1417-1424). Studies evaluating risk of cerebral palsy following maternal fever or infection were not included in the metaanalysis, a factor possibly accounting for the different conclusion vs the Canadian study.

In an editorial, Linda de Vries (Wilhelmina Children's Hospital, Utrecht, the Netherlands) expands on measurement of cytokines in newborns with WMI, and the finding that chorioamnionitis with or without funisitis makes a very low birth weight infant more susceptible to hypotension at time of birth. (Ann Neurol 2009;66:127-129).

\title{
EARLY EEG FINDINGS AND HI-ENCEPHALOPATHY OUTCOMES
}

The value of the EEG as a predictor of outcome in term infants with hypoxicischemic encephalopathy (HIE) was determined in a study at Cork University Maternity Hospital and St Vincent's University Hospital, Dublin, Ireland. Continuous video-EEG was recorded from $<6$ hours to 72 hours after delivery. One-hour EEG segments at 6, 12, 24, and 48 hours of age were analyzed visually, and neurologic outcome was assessed at 24 months. Of 44 infants who completed follow-up, 20 (45\%) had abnormal neurodevelopmental outcomes. Clinical Sarnat scoring at 24 hours classified 18 infants with grade I HIE, 17 with grade II, and 9, grade III. EEG abnormalities were greatest on the earliest recordings of all cases and improved with time. Best predictive ability occurred at 6 hours of age. Normal/mildly abnormal EEG at 6, 12, or 24 hours had $100 \%$ positive predictive values for normal outcome, and negative predictive values of $67 \%$ to $76 \%$. At 24 hours, the number of infants assigned to each EEG grade was 6 normal, 11 moderately abnormal, 9 severe, and 3 isoelectric. Background amplitude of $<30 \mathrm{mcV}$, interburst interval of $>30 \mathrm{sec}$, electrographic seizures, and absence of sleep-wake cycling at 48 hours were associated with abnormal outcome. Normal EEG within 6 hours after birth was associated with normal neurodevelopment at 24 months. (Murray DM, Boylan GB, Ryan CA, Connolly S. Early EEG findings in hypoxic-ischemic encephalopathy predict outcomes at 2 years. Pediatrics Oct 2009;124:e459-e467). (Respond: Deidre M Murray MD PhD, Department of Pediatrics and Child Health, Clinical Investigation Unit, Cork University Hospital, Wilton, Cork. Ireland. E-mail: d.murray@ucc.ie).

COMMENT. Early EEG is a reliable predictor of neurodevelopmental outcome in term infants with HIE. EEG abnormalities evolving in the first 48 hours of life predict a poor outcome, and normal EEG at 6 hours of age is predictive of a normal outcome at 2 years. Early EEG study at 6 to 12 hours and repeat study at 48 hours should predict outcomes successfully in $95 \%$ of cases. EEG seizures detected by continuous monitoring correlate with poor outcome.

Neonatal EEG in periventricular leukomalacia (PVL). In a study at Anjo Kosei Hospital and other centers in Japan, EEG findings varied with the severity of PVL (noncystic, localized cystic, and extensive cystic) and the timing of recording. To detect PVL, $>2$ EEG recordings are recommended, 1 within 48 hours after birth for acute stage abnormalities, and 
1 in the second week to detect chronic stage abnormalities. (Kidokoro $\mathrm{H}$ et al. Pediatrics Oct 2009;124:e468-e475). (E-mail: kidokoro@kosei.anjo.aichi.jp).

Dr Joseph J Volpe, in an editorial, comments that the report by Kidokoro and associates shows that the EEG may be important in diagnosis, timing, and severity of PVL (Pediatrics Oct 2009;124:e542-e544), but the infants in this study were a severely affected subset of the premature population. In general, MRI in the neonatal period is the most effcctive method of identifying white-matter injury in premature infants. Ultrasonography is useful in detection of severe injury. The timing of the insult by EEG is useful in the decision to order potentially protective interventions such as antioxidants etc.

\section{EFFECT OF NEONATAL SEIZURES ON COGNITIVE OUTCOME OF HYPOXIC-ISCHEMIC ENCEPHALOPATHY}

The independent effect of clinical neonatal seizures and their treatment on longterm neurodevelopmental outcome in 77 term newborns at risk for hypoxic-ischemic encephalopathy (HIE) was determined in a study at University of California San Francisco. Clinical seizures were recorded and graded prospectively assigning points $(0-10)$ for frequency, status, medications, and EEG abnormal background and epileptiform discharges. Eleven children (14.3\%) had severe neonatal seizures (composite seizure score $>4$ ), 14 (18.2\%) had mild/moderate seizures (score 1 to 3 ), and $52(67.5 \%)$ had no seizures (seizure score 0 ). Of the 25 infants with seizures, EEG was abnormal in 14 (56\%). The severity of HIE measured by MRI was most highly associated with cognitive outcome, measured by WPPSI-R and neuromotor score, at age 4 years. The pattern of the HIE correlated with severity of seizures $(\mathrm{P}<0.0001)$; basal nuclei predominant patern was associated with severe seizures, and the watershed pattern with mild/moderate seizures. Children with severe seizures had a lower FSIQ than those with mild/moderate seizures $(\mathrm{P}<0.0001)$. (Glass HC, Glidden D, Jeremy RJ, Barkovich AJ, Ferriero DM, Miller SP. Clinical neonatal seizures are independently associated with outcome in infants at risk for hypoxic-ischemic bnrain injury. J Pediatr September 2009;155:318-323). (Respond: Hannah C Glass MDCM, University of California San Francisco, Department of Neurology, Box 0663, 521 Parnassus Ave, C-215, San Francisco, CA 94143. E-mail: Hannah.Glass@ucsf.edu).

COMMENT. The authors conclude that clinical neonatal seizures with birth asphyxia are associated with worse neurodevelopmental outcome, independent of the severity of hypoxic-ischemic brain injury. The effect of the seizures themselves could not be differentiated from the cognitive effects of treatment with phenobarbital and phenytoin, however. Also, almost half of patients with clinical seizures had a normal EEG. Seizure severity was graded by frequency, medication use, and EEG, but not by seizure pattern. According to Volpe JJ (N Engl J Med 1973;289:413; also, In Gluck L. Editor. Intrauterine Asphyxia and the Developing Fetal Brain. Chicago. Year Book Med Pub, 1977), virtually all infants with HIE related seizures have "subtle" seizures. Subtle seizures are manifested by 1) tonic horizontal deviation or jerking of eyes; 2) eye-lid blinking or fluttering; 3) sucking, smacking of lips; 4) 'swimming" or "rowing" movements of limbs; and/or 5) apneic spells. Infants also exhibit multifocal clonic seizures or decerebrate/decorticate tonic seizures in 